\title{
Addition of simvastatin to the standard therapy increases survival and is safe in patients with decompensated cirrhosis
}

\author{
Alberto E Muñoz ${ }^{1,3 *}$, Walter Taddey ${ }^{2}$ and Pablo Salgado ${ }^{3}$ \\ ${ }^{1}$ Hospital Dr. Carlos B. Udaondo, Av. Caseros 2061, PC 1264, City of Buenos Aires, Argentina \\ ${ }^{2}$ Coronary Unit, José de San Martín Clinic Hospital, University of Buenos Aires, Buenos Aires, Argentina \\ ${ }^{3}$ Institute of Public Health Research, School of Dentistry, University of Buenos Aires, Buenos Aires, Argentina
}

\begin{abstract}
Background: Death by cardiovascular events has reduced by statins due to altering atherosclerosis development. As of 2007 , no data on the use of statins in patients with decompensated cirrhosis were available.
\end{abstract}

Aims: To evaluate the simvastatin efficacy and safety in patients with decompensated cirrhosis and cardiovascular factors.

Methods: We performed a matched cases-series study. The case group included patients who agreed to add simvastatin to the standard therapy. The series group included patients who did not accept to add this drug to the standard of care. Each group had nine patients. Age, gender, cirrhosis etiology, Child-Pugh class, and Model for End-Stage Liver Disease (MELD) score matched case and series group in a ratio 1:1.

Results: The intervals between cirrhosis complications in the case and series groups were $33.6 \pm 19.9$ months and $9.4 \pm 8.2$ months, respectively, $P=0.0065$. There was a significant deterioration of the liver function, which was evaluated through Child-Pugh and MELD scores in the series group while it was not affected in the case group. Median survival in the case group was 107 months, whereas it was 20 months in the series group $(\mathrm{HR}=0.14 ; P<0.0001)$. On the other hand, no patient in the case group experienced simvastatin-related adverse events. Furthermore, no patient in the case or series groups developed cardiovascular events.

Conclusions: The addition of simvastatin to the standard therapy in patients with decompensated cirrhosis and cardiovascular risk factors was efficient as it decreased the patient's mortality. Furthermore, the simvastatin was safe as patients showed good tolerance, considering that they did not develop adverse effects or serious adverse effects.

\section{Introduction}

Portal hypertension is mainly determined by the morphological changes occurring in chronic liver diseases [1]. This is aggravated by a "dynamic component" due to the active - reversible - contraction of different elements of the porto-hepatic bed [1]. The primary determinant of the "dynamic component' is a decreased synthesis of nitric oxide (NO) in the intrahepatic circulation [1]. As a consequence of portal hypertension complications, patients with decompensated cirrhosis have a high mortality rate [2].

On the other hand, atherosclerosis has been widely recognized as an inflammatory process that leads to acute clinical cardiovascular events such as unstable angina, myocardial infarction, and/or cerebral stroke [3]. Patients with cirrhosis may suffer from these disorders. A nationwide cohort study in Denmark included 10.154 cirrhotic patients [4]. After one year of follow-up, the patients died from no liver diseases. Ischemic heart disease, peripheral vascular disease, and cerebrovascular disease were the leading causes of death.

In addition, statins prevent atherosclerotic inflammatory vascular disease by two mechanisms [5]. First, a direct mechanism that works through the reduction of the plasma cholesterol level. Second, there is an indirect mechanism known as "pleiotropic effect." The latter mechanism is independent of cholesterol as the reduction of these events is independent of the serum cholesterol level and the isolated cholesterol lowering. Pleiotropic effects include NO enhancement bioavailability in endothelial cells [6].

Finally, Zafra et al. [7] have demonstrated that simvastatin administration to patients with cirrhosis and portal hypertension decreased hepatic sinusoidal resistance by increasing NO output.

Considering the information available as of 2007, one of us (AEM) offered to administrate simvastatin to patients with decompensated cirrhosis and cardiovascular risk factors to assess its effectiveness and safety.

${ }^{\star}$ Correspondence to: Alberto E. Muñoz, Hospital Dr. Carlos B. Udaondo, Av. Caseros 2061, PC 1264, City of Buenos Aires, Argentina, Tel: +54-9-11-41770351; Fax: 54-4306-2033; E-mail: aemunoz@intramed.net

key words: cirrhosis, cardiovascular risk factors, statins, safety, survival

Received: October 04, 2019; Accepted: October 18, 2019; Published: October 21,2019 


\section{Materials and Methods}

\section{Study design and place of study}

After obtaining the authorization from the Medical Management of Clínica Bazterrica and the consent of the patients or their family, we assessed all medical records of patients with decompensated and cirrhosis cardiovascular risk factors.

Clínica Bazterrica is a 165 -bed tertiary care adult clinic. It serves the adult population of the City of Buenos Aires, Argentina. In this medical center, patients receive medical care on an outpatient and an inpatients basis.

This matched cases-series study was conducted from April 2007 to October 2019. We followed the STROBE Statement to design and write this study [8].

\section{Patients}

Case group: All patients who agreed to receive simvastatin in addition to the standard therapy were included in this group. Before including the patients in this group, the simvastatin potential beneficial effects and risks for cirrhosis were explained to them. The patients should have met the following inclusion criteria: to be over 18 years, previous diagnosis of decompensated cirrhosis, documented existence of cardiovascular risk factors, absence of pregnancy, and not being candidates for liver transplantation.

Series group: The patients who did not agree to receive simvastatin to the standard therapy were included in this group. They met the same criteria as the case group.

The following parameters matched both groups: age \pm 3 years, gender, etiology of cirrhosis, Child-Pugh class, and Model for EndStage Liver Disease (MELD) score \pm 3 points. The groups matched at a ratio of 1:1.

\section{Proceedings}

The data analyzed at the initial visit included demographic, clinical, biochemical, ultrasound, and endoscopic parameters. Moreover, Child-Pugh class, Child-Pugh score, and MELD score were assessed as well. The data analyzed in the following medical appointments during the study were the same as at the initial interview.

The simvastatin dose ranged from 10 to $40 \mathrm{mg} / \mathrm{day}$. This dose range is the recommended dose to treat hypercholesterolemia [9]. Dose reduction or transitory therapy interruption was applied in the case of simvastatin adverse events. In the same way, simvastatin was permanently withdrawn due to serious adverse events.

\section{Efficacy and safety end points}

The efficacy of simvastatin was measured by analyzing the proportion of patients with cirrhosis complications, cardiovascular events, and death during the whole study.

Furthermore, simvastatin's safety was assessed through the proportion of patients with adverse events and serious adverse events.

\section{Statistical analysis}

The data were collected, classified, and analyzed using SPSS Statistics 25.0 statistical package program (IBM). Figure 1 was designed with MedCalc 11.2 version. The date of the first cirrhosis complication was established as the time zero for both groups. A comparison of dichotomous variables was made by McNemar test. Paired t-test was used to compare continuous variables. In the case of abnormally distributed variables, non-parametric Wilcoxon-signed rank test was implemented. The cumulative survival function was estimated by the Kaplan-Meier method. Both groups were compared with the stratified log-rank test and hazard ratios (HRs) (95\% confidence interval [CI]), calculated using the Cox model. A $P<0.05$ was considered statistically significant.

\section{Results}

The matching and inclusion criteria of the case group and the series group are shown in table 1. Age, gender, etiology of cirrhosis, ChildPugh class, and MELD score matched the series and cases patients. Therefore, both groups were similar.

Each group had nine patients. The mean age was 63 years (range was from 48 to 81 years) in the case group. On the other hand, the mean age was 66 years (range was from 46 to 83 years) in the series group. Six out of nine patients were female in each group. Five patients in both groups had HCV. The other four patients in each group had alcohol, primary biliary cholangitis, autoimmune hepatitis, and nonalcoholic steatohepatitis as the etiology of cirrhosis. Ascites was the first most common complication of cirrhosis in both groups. Moreover, the most common cardiovascular risk factor was diabetes. The main reason for no liver transplantation was the relatively proper liver function, Child-Pugh A or B, and MELD $<15$.

Table 2 displays the baseline biochemical parameters and findings during the study of the case and series groups. There was no difference in the biochemical parameters between both groups, as probably many of them matched the case and the series groups. On the other hand, HCV patients were relapsers or non-responders to PegIFN plus ribavirin or non-treated due to decompensated cirrhosis. Likewise, hepatocellular carcinoma rate was higher in the case group than in a series group, probably related to the longer follow-up of the former group.

The case group was followed up for $87.0 \pm 50.8$ months, and the series group was followed up for $24.2 \pm 19.9$ months, $P=0.012$. During the follow-up period, the study showed that the interval between cirrhosis complications in the case group was $33.6 \pm 19.9$ months versus the series group, $9.4 \pm 8.2$ months, $\mathrm{P}=0.0065$. Moreover, the liver function deterioration was evaluated through Child-Pugh score and MELD score at the end of the study versus baseline. In the case group, Child-Pugh scores were $8.4 \pm 3.1$ versus $6.8 \pm 1.4, \mathrm{P}=0.1786$, and MELD scores were $14.6 \pm 8.7$ versus $10.8 \pm 4.9, \mathrm{P}=0.0983$, respectively. In the series group, Child-Pugh scores were $10.9 \pm 2.6$ versus $7.2 \pm 1.9$, $\mathrm{P}=0.0026$ and MELD scores were $21.7 \pm 7.9$ versus $11.8 \pm 4.8, \mathrm{P}=$ 0.0028 , respectively.

At the end of the study, four out of nine patients in the case group, which was $44 \%$ of the group, and all the patients in the series group died. Table 2 displays the causes of death of each group. The median survival in the case group was 107 months, whereas it was 20 months in the series group $(\mathrm{HR}=0.14$; 95\% CI: 0.04-0.49; stratified log-rank test, $P<0.0001$ ) (Figure 1).

As a result of this study, any patient experienced simvastatinrelated adverse events or serious adverse events. Furthermore, the serum aminotransferases and/or creatine kinase concentration were not increased. Therefore, there was no need to reduce the dose of simvastatin in any patient in the case group, nor to interrupt temporarily, or to discontinue simvastatin administration. No patient in the case group or the series group developed cardiovascular events. 
Table 1. Matching and inclusion criteria of the case group and the series group

\begin{tabular}{|c|c|c|}
\hline Variable & $\begin{array}{c}\text { Case group } \\
n=9\end{array}$ & $\begin{array}{l}\text { Series group } \\
\quad \mathbf{n}=\mathbf{9}\end{array}$ \\
\hline Age, years, mean, (SD) & $63(11)$ & $66(9)$ \\
\hline Gender, female, n (\%) & $6(66)$ & $6(66)$ \\
\hline \multicolumn{3}{|l|}{ Etiology of cirrhosis, $n$} \\
\hline Hepatitis $\mathrm{C}$ virus & 4 & 4 \\
\hline Hepatitis C virus plus alcohol & 1 & 1 \\
\hline Alcohol & 1 & 1 \\
\hline Primary biliary cholangitis & 1 & 1 \\
\hline Autoimmune hepatitis & 1 & 1 \\
\hline Non-alcoholic steatohepatitis & 1 & 1 \\
\hline Child-Pugh class, A /B / C, n & $4 / 4 / 1$ & $4 / 4 / 1$ \\
\hline MELD score, mean, (SD) & $10.8(4.9)$ & $11.8(4.8)$ \\
\hline$D^{\prime}$ Amico stage, 3/4/5, n & $2 / 4 / 3$ & $1 / 8 / 0$ \\
\hline \multicolumn{3}{|l|}{ Cardiovascular risk factors ${ }^{\mathrm{a}}, \mathbf{n}$} \\
\hline Family history of premature ASCVD & 2 & 2 \\
\hline Diabetes & 5 & 4 \\
\hline Hypertension & 4 & 2 \\
\hline Obesity & 2 & 3 \\
\hline Metabolic syndrome & 2 & 2 \\
\hline Current smoking & 3 & 3 \\
\hline Hypercholesterolemia & 1 & 1 \\
\hline \multicolumn{3}{|c|}{ Cardiovascular risk parameters, ranges } \\
\hline Glucose, $\mathrm{mg} / \mathrm{dL}$ & $130-135$ & $132-148$ \\
\hline LDL cholesterol, mg/dL & 210 & 220 \\
\hline Blood pressure, $\mathrm{mmHg}$ & $140 / 74-144 / 78$ & $142 / 70-146 / 74$ \\
\hline Body mass index, $\mathrm{kg} / \mathrm{m}^{2}$ & $31.2-32.7$ & $30.5-33.3$ \\
\hline Smoking pack-year & Oct-15 & Oct-15 \\
\hline \multicolumn{3}{|l|}{ No liver transplantation, reasons, $n$} \\
\hline Child-Pugh A or B + MELD $<15, \mathrm{n}$ & 8 & 8 \\
\hline Age $>75$ years old, $n$ & 2 & 2 \\
\hline Active alcoholism, $\mathrm{n}$ & 1 & 1 \\
\hline Died in the waiting list, $\mathrm{n}$ & 1 & 1 \\
\hline Refused, $\mathrm{n}$ & 1 & 1 \\
\hline
\end{tabular}

${ }^{a}$ Some patients had more than one cardiovascular risk factor. SD: Standard Deviation; MELD: Model for End-Stage Liver Disease; ASCVD: Atherosclerotic Cardiovascular Disease. P values not applicable for age, gender, etiology of cirrhosis, Child-Pugh class, and MELD score because these parameters matched case group with series group. All other P values are $>0.05$.

Table 2. Baseline biochemical parameters and study findings of the case group and the series group

\begin{tabular}{|c|c|c|}
\hline Parameters/findings & $\begin{array}{c}\text { Case group } \\
\quad \mathrm{n}=9\end{array}$ & $\begin{array}{c}\text { Series group } \\
\mathbf{n}=\mathbf{9}\end{array}$ \\
\hline Bilirubin mg/dL, mean, (SD) & $1.6(1.2)$ & $1.7(1.2)$ \\
\hline AST IU/L, mean, (SD) & $101(82)$ & $90(48)$ \\
\hline ALT IU/L, mean, (SD) & $67(47)$ & $62(24)$ \\
\hline Albumin g/dL, mean, (SD) & $3.4(0.4)$ & $3.2(0.4)$ \\
\hline Prothrombin time $\%$, mean, (SD) & $74(20)$ & $72(19)$ \\
\hline INR, mean, (SD) & $1.3(0.3)$ & $1.3(0.4)$ \\
\hline Creatinine mg/dL, mean, (SD) & $0.8(0.3)$ & $0.9(0.4)$ \\
\hline CK IU/L, mean, (SD) & $83(31)$ & N.A. \\
\hline \multicolumn{3}{|l|}{ HCV treatment, $n$} \\
\hline Relapsers to Peg-IFN + RBV & 1 & 1 \\
\hline Non-responders to Peg-IFN + RBV & 1 & 2 \\
\hline Cured with DAA & 1 & 0 \\
\hline No due to decompensated cirrhosis & 2 & 2 \\
\hline \multicolumn{3}{|l|}{ Hepatocellular carcinoma (BCLC), n } \\
\hline Early & 3 & 0 \\
\hline Terminal & 0 & 1 \\
\hline \multicolumn{3}{|l|}{ Cause of death, $n$} \\
\hline Liver failure & 2 & 4 \\
\hline Variceal bleeding & 1 & 2 \\
\hline Spontaneous bacterial peritonitis & 1 & 1 \\
\hline Hepatocellular carcinoma & 0 & 1 \\
\hline Sepsis & 0 & 1 \\
\hline
\end{tabular}

AST: Aspartate Aminotransferase, normal range: 0-50 IU/L; ALT: Alanine Aminotransferase, normal range: 0-50 IU/L; INR: International Normalized Ratio; CK: Creatine Kinase, normal range: 0-171 IU/L; N.A.: Not Available; HCV: Hepatitis C Virus; Peg-IFN: Pegylated-Interferon; RBV: Ribavirin; DAA: Direct-Acting Antivirals; BCLC: Barcelona Clinic Liver Cancer stages. All $\mathrm{P}$ values are $>0.05$. 


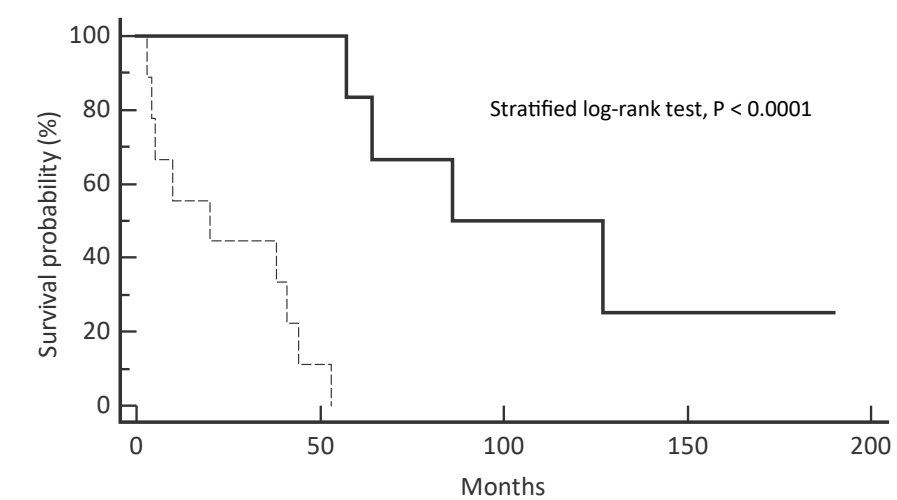

Number at risk

Group: 1

Group: $2^{9}$

9

7

3

1

1

1

0

0

0

Figure 1. Kaplan-Meier curve which shows that patients in the case group (continuous line; group 1, bottom) had significantly higher survival than patients in the series group (dashed line; group 2, bottom)

\section{Discussion and conclusion}

This matched cases-series study showed that the addition of simvastatin to the standard therapy in patients with decompensated cirrhosis and cardiovascular risk factors showed an extended survival in the case group. Furthermore, simvastatin administration was safe, as it did not cause adverse effects and/or serious adverse effects. No patient in any group developed cardiovascular events during the study.

The high mortality rate of decompensated cirrhosis stresses the need for other therapies. Treatments used in patients with cardiovascular diseases could be an option for patients with decompensated cirrhosis [10]. Therefore, cirrhosis could be treated cost-effectively by combined therapies with authorized, economical, and relatively safe medications, including simvastatin [11].

In addition, recent studies have established the relationship between cardiovascular disease and liver disease, which is mainly associated with HCV [12-15]. It should be highlighted that all patients in the present study had a cirrhosis diagnose. Moreover, five out of nine patients in the case group had HCV, which was associated with cardiovascular risk factors.

Regarding the efficacy of simvastatin, the first description in this study was the longer follow-up period in patients who agreed to take simvastatin versus those who did not take it. Respecting this finding, Kaplan et al., in a retrospective cohort study in veterans with a new diagnosis of cirrhosis, observed that each cumulative year of statin exposure was associated with an independent decrease in mortality of patients with cirrhosis Child-Pugh A and B [16].

Bearing in mind that the main finding of this study is the higher survival in patients who took simvastatin compared to those who did not take it, Cabrera et al. [17] suggested that statins could improve the survival of decompensated cirrhosis by two mechanisms. The first mechanism is by reducing the impact of cirrhosis complications on the liver function. The Child-Pugh score and MELD score in the present study showed that there was a significant deterioration of the liver function in those patients who did not take simvastatin in comparison with those patients who agreed to add simvastatin to the standard therapy. Moreover, Pollo-Flores et al. [18] observed that a simvastatin treatment to evaluate its effects on hepatic venous pressure gradient was associated with a slight improvement of the liver function, which was assessed through the Child-Pugh score. The other mechanism suggested by Cabrera et al. is by preventing cirrhosis complications [17]. According to the present study, the interval between the cirrhosis complications increased in those patients who agreed to add simvastatin to the standard therapy in comparison to those who did not take it. Therefore, it was understood that simvastatin prevents cirrhosis complications by extending the interval period between cirrhosis complications. Overall, the increased survival showed in this study could be supported by both mechanisms proposed by Cabrera et al. based on the pleiotropic effects of simvastatin. Lastly, the results of the largest randomized controlled trial which assessed the effects of simvastatin in cirrhosis should be considered [19]. The patients included in this trial had cirrhosis and recovered from an acute variceal bleeding episode. The BLEPS (Bleeding Prevention with Simvastatin) trial showed that the addition of simvastatin to the standard of care did not reduce rebleeding, but improved survival in patients with ChildPugh class A or B cirrhosis.

The second end point of this study was simvastatin safety, taking into account statins pharmacokinetic alterations showed in patients with cirrhosis [20]. The fact that no dose reduction and/or the lack of simvastatin transitory interruption by adverse events or discontinue simvastatin by serious adverse events, particularly by muscle and/ or liver injuries, proved that simvastatin would be a safe drug to administrate in patients with decompensated cirrhosis Child-Pugh A or B. These results would be complementary to those of the LiverHope-Safety trial [21]. They observed a significant increase in adverse events in Child-Pugh C patients treated with simvastatin $40 \mathrm{mg} /$ day.

Finally, in the absence of mature data from observational studies in large populations of patients with cirrhosis [22], a matched case-series study has several advantages compared with a simple retrospective descriptive report. In the present study, each case was intentionally matched to a series based on five criteria that are potentially essential variables in determining outcomes. The case and series groups were contemporaneous which allowed to have a better control of the potential mismanagement of these patients, including the simvastatin dose, adverse events and complications of cirrhosis. Even though the present study could not reduce potential biases compared to a simple retrospective descriptive report, it could improve the understanding of the addition of simvastatin to the standard of care in patients with decompensated cirrhosis.

In conclusion, the addition of simvastatin to the standard therapy in patients with decompensated cirrhosis and cardiovascular risk factors is statistically significant, and above all, clinically relevant in comparison to a standard treatment as it improved survival and simvastatin was well-tolerated by the patients.

\section{References}

1. Bosch J, Garcia-Pagan JC (2000) Complications of cirrhosis. I. Portal hypertension. $J$ Hepatol 32: 141-156. [Crossref]

2. D'Amico G, Garcia-Tsao G, Pagliaro L (2006) Natural history and prognostic indicators of survival in cirrhosis: A systematic review of 118 studies. $J$ Hepatol 44: 217-231. [Crossref]

3. Veillard NR, Mach F (2002) Statins: the new aspirin? Cell Mol Life Sci 59: 1771-1786. [Crossref]

4. Sorensen HT, Thulstrup AM, Mellemkjar L, Jepsen P, Christensen E, et al. (2003) Long-term survival and cause-specific mortality in patients with cirrhosis of the liver: a nationwide cohort study in Denmark. J Clin Epidemiol 56: 88-93. [Crossref]

5. Liao JK, Laufs U (2005) Pleiotropic effects of statins. Annu Rev Pharmacol Toxicol 45: 89-118. [Crossref] 
6. Wolfrum S, Jensen KS, Liao JK (2003) Endothelium-dependent effects of statins. Arterioscler Thromb Vasc Biol 23: 729-736. [Crossref]

7. Zafra C, Abraldes JG, Turnes J, Berzigotti A, Fernández M, et al. (2004) Simvastatin enhances hepatic nitric oxide production and decreases the hepatic vascular tone in patients with cirrhosis. Gastroenterology 126: 749-755. [Crossref]

8. Vandenbroucke JP (2007) The making of STROBE. Epidemiology 18: 797-799. [Crossref]

9. Plosker GL, Simvastatin MD (1995) A reappraisal of its pharmacology and therapeutic efficacy in hypercholesterolaemia. Drugs 50: 334-363. [Crossref]

10. Hingorani AD, Hemingway $\mathrm{H}$ (2011) How should we balance individual and population benefits of statins for preventing cardiovascular disease? BMJ 342: c6244. [Crossref]

11. Tsochatzis EA, Bosch J, Burroughs AK (2010) Prolonging survival in patients with cirrhosis: old drugs with new indications. Gastroenterology 139: 1813-1815. [Crossref]

12. Kalaitzakis E, Rosengren A, Skommevik T, Björnsson E (2010) Coronary artery disease in patients with liver cirrhosis. Dig Dis Sci 55: 467-475. [Crossref]

13. Targher G, Bertolini L, Padovani R, Rodella S, Arcaro G, et al. (2007) Differences and similarities in early atherosclerosis between patients with non-alcoholic steatohepatitis and chronic hepatitis B and C. J Hepatol 46: 1126-1132. [Crossref]

14. Goossens N, Negro F (2017) Cardiovascular manifestations of hepatitis C virus. Clin Liver Dis 21: 465-473. [Crossref]

15. Lee KK, Stelzle D, Bing R, Anwar M, Strachan F, et al. (2019) Global burden of atherosclerotic cardiovascular disease in people with hepatitis $\mathrm{C}$ virus infection: systematic review, meta-analysis, and modelling study. Lancet Gastroenterol Hepatol 4: 794-804. [Crossref]
16. Kaplan DE, Serper MA, Mehta R, Fox R, John B, et al. (2019) Effects of hypercholesterolemia and statin exposure on survival in a large national cohort of patients with cirrhosis. Gastroenterology 156: 1693-1706. [Crossref]

17. Cabrera L, Abraldes JG (2016) Statins: the panacea of cirrhosis? Curr Hepatology Rep 15: $1-7$.

18. Pollo-Flores P, Soldan M, Santos UC, Kunz DG, Mattos DE, et al. (2015) Three months of simvastatin therapy vs. placebo for severe portal hypertension in cirrhosis: a randomized controlled trial. Dig Liver Dis 47: 957-963. [Crossref]

19. Abraldes JG, Villanueva C, Aracil C, Turnes J, Hernandez-Guerra M, et al. (2016) Addition of simvastatin to standard therapy for the prevention of variceal rebleeding does not reduce rebleeding but increases survival in patients with cirrhosis. Gastroenterology 150: 1160-1170. [Crossref]

20. Bellosta S, Paoletti R, Corsini A (2004) Safety of statins. Focus on clinical pharmacokinetics and drug interactions. Circulation 109: 11150-11157. [Crossref]

21. Pose E, Napoleone L, Amin A, Campion D, Jimenez C, et al. (2019) Safety of two different doses of simvastatin plus rifaximin in decompensated cirrhosis (LIVERHOPESAFETY): a randomised, double-blind, placebo-controlled, phase 2 trial. Lancet Gastroenterol Hepatol 5: 31-41. [Crossref]

22. Kamal S, Khan MA, Seth A, Cholankeril G, Gupta D, et al. (2017) Beneficial Effects of statins on the rates of hepatic fibrosis, hepatic decompensation, and mortality in chronic liver disease: A systematic review and meta-analysis. Am J Gastroenterol 112: 1495-1505. [Crossref]

Copyright: (C2019 Muñoz AE. This is an open-access article distributed under the terms of the Creative Commons Attribution License, which permits unrestricted use, distribution, and reproduction in any medium, provided the original author and source are credited. 